\title{
GENERALIZED FRAGILITY CURVES FOR CONCRETE COLUMNS EXPOSED TO FIRE THROUGH SURROGATE MODELLING
}

\author{
Ranjit Kumar Chaudhary ${ }^{1}$, Balša Jovanović ${ }^{2}$, Thomas Gernay ${ }^{3}$, Ruben Van Coile ${ }^{4}$
}

\begin{abstract}
Common structural fire design relies on recommendations from design codes, or (a single or small set of) more advanced numerical analyses. When applying such procedures to the design of structures under normal loading conditions, adequate safety is ensured through calibrated safety factors and ample experience with structural failures. This is however not the case when considering accidental fire loading, where the stochasticity in the structural fire behaviour is rarely fully acknowledged. Therefore, a significant interest in the use of probabilistic approaches to evaluate structural fire performance, which take into account the uncertainty associated with model parameters, can be observed among researchers, with a special focus on the development of fragility curves. The calculation of fragility curves is, however, a laborious task, demanding huge computational expense, mainly attributed to the adoption of advanced calculation procedures and the need for a large number of model evaluations. The present study contributes to addressing the limitations imposed by these computational requirements through the development of surrogate models for fire exposed structural members. To achieve this, a framework for carrying out probabilistic studies of structures under fire through the use of surrogate modelling is presented. The framework is applied to a concrete column subjected to a standard fire and proves efficiency and accurateness for the selected simple example. Future studies will investigate the applicability of the framework to structural assemblies under physically-based fires.
\end{abstract}

Keywords: Fire safety; probabilistic approach; surrogate modelling; concrete columns; machine learning.

\section{INTRODUCTION}

Traditionally, the structural fire design is carried out based on prescriptive guidelines and code-based recommendations. These recommendations refer to a standard building type, in a specific region, serving a specific purpose, which undermines the idea of its application to a wide range of structural configurations. Thus, the structural fire design based on traditional approaches appears to result in case-dependent outcomes in terms of prescribed safety level. To demonstrate adequate structural fire safety, whether for developing more uniform guidance or for designing exceptional structures, the risk profile for the structures should explicitly be considered. When evaluating the risk profile, all the influential conditions of possible fires and their probabilities, together with the uncertainty associated with structural system, are taken into account [1-2]. Acknowledging this, there has recently been an increase in the use of probabilistic methodologies for structural fire design. Several probabilistic frameworks can be found in the literature for

\footnotetext{
${ }^{1}$ Ph.D. researcher, Ghent University, Belgium.

e-mail: ranjitkumar.chaudhary@ugent.be, ORCID: https://orcid.org/0000-0002-1104-5859 2

Ph.D. researcher, Ghent University, Belgium.

e-mail: balsa.jovanovic@ugent.be, ORCID: https://orcid.org/0000-0001-5200-5848

${ }^{3}$ Assistant professor, Johns Hopkins University, United States.

e-mail: tgernay@jhu.edu, ORCID: https://orcid.org/ 0000-0002-3511-9226

${ }^{4}$ Assistant Professor, Ghent University, Belgium.

e-mail: ruben.vancoile@ ugent.be, ORCID: https://orcid.org/ 0000-0002-9715-6786

https://doi.org/10.14264/45b645e
} 
probabilistic assessment of fire exposed structures. For example, Shrivastava et al. [3] applied performance based earthquake engineering (PBEE) framework for probabilistic studies in structural fire engineering (SFE), where annual rates of exceedance of hazard intensity and structural response were estimated. At the same time, Heidari et al. [4] estimated the failure probability of a fire exposed concrete slab to assess its reliability adopting a Monte-Carlo (MC) method, and Gernay et al. [5] developed fragility curves to assess the performance of steel building under fire hazard. Out of the available probabilistic approaches, the development of fragility curves has been found to be popular among researchers as it is conceptually easy to understand and allows faster design iterations [5].

Although available probabilistic methodologies, such as the development of fire fragility curves, can contribute to enable a safe and reliable design for fire exposed structures [2-6], their implementation has been found arduous and computationally challenging. The commonly large computational demand can mainly be attributed to the multi-step calculation procedure required for fire analysis of structures, together with the need for a large number of structural evaluations for probabilistic estimations. For instance, the Monte Carlo approach adopted for reliability assessment of reinforced concrete (RC) slabs by Van Coile et al. [6] involves about 10,000 structural evaluations, demonstrating substantial computational demand. A similar difficulty can be observed in the probabilistic studies carried out by Guo et al., and Hopkin et al. [78] for the probabilistic evaluation of protected steel elements. To address this limitation, some studies adopt simplified structural fire models (simply supported beam considered by Guo et al.; uniform steel temperature assumption by Hopkin et al.), while others limit the number of structural evaluations through efficient stochastic modelling procedures [5,7]. Although these more advanced approaches managed to reduce the computational expense in the above cases, their execution requires careful error analysis and added expert knowledge. Furthermore, the above-mentioned probabilistic studies have a drawback that they are limited to a specific case, where any alteration in the design parameter involves re-assessment of the entire probabilistic evaluation.

Therefore, there is a need for a technique which can reduce the computational effort significantly and allow quick iterations for alteration in design parameters. In this regard, Machine learning (ML) algorithms, which can mimic a complex high-fidelity model [9] (here, SFE models), could be a promising technique for probabilistic analysis of structures in fire. Additionally, such models allow rapid generation of fragility curves at limited computational expense when design parameters are altered (within the range of applicability). Recent applications of ML algorithms in the field of fire safety engineering can be found in Dexters et al. [10], where lasso-regression based surrogate models were adopted to predict the flashover in a compartment. Likewise, Naser [11-12] carried out a series of investigations based on ML algorithms in the field of SFE. Based on this available literature, it can be hypothesized that ML algorithms can efficiently be used to predict the response of fire exposed structures and thereby allow computationally efficient probabilistic analysis for SFE models.

The present study intends to present a computationally efficient methodology for probabilistic analysis of a fire exposed structure through the use of ML algorithms. The methodology is demonstrated through application to a RC column under standard fire exposure. The structure of the paper is as follows. Initially the methodology for development of surrogate model is discussed (Section 2). Section 3 presents the considered fire exposed column and the development of the surrogate model. Finally, the probability distribution functions based on actual and surrogate models are evaluated and compared in Section 4. Section 4 further demonstrates the application of the surrogate model for developing generalized fragility curves applicable to design changes with respect to the ISO834 exposure duration.

\section{METHODOLOGY: SURROGATE MODELING}

The surrogate models developed through ML algorithms can be found in a wide variety of fields for evaluating the response of physical systems $[910,13]$. Similarly, in the field of SFE, ML algorithms such as regression, GA and ANN have demonstrated promising performance [10-12]. However, the use of ML techniques in fire safety engineering remains hardly explored. In this regard, the current study explores the potential of regression based surrogate models for approximating the response of SFE models. Regression based surrogate models are simple and interpretable compared to algorithms such as ANN and GA and thus 
allow to evaluate the effect of model parameters on the response of the physical system. Here, polynomial regression-based surrogate models are adopted to predict the response of fire exposed structures. The methodology for the development of a regression-based surrogate model is discussed hereafter.

\subsection{Training data generation}

The first step for the training data generation is the identification of model variables on which the response of the high-fidelity model depends. If $x=\left[x_{1}, x_{2}, x_{3}, \ldots \ldots \ldots \ldots x_{\mathrm{r}-1}, x_{\mathrm{r}}\right]$ represents a vector of model variables, then the response, $y$ of the high-fidelity model, $h(x)$ is given by:

$$
y=h(x)
$$

A sampling scheme for the considered parameters is then developed (e.g. Latin hypercube sampling (LHS) can be used [14]), accounting for a range of possible design configurations. Finally, the response of the high-fidelity model, i.e. in this case the finite element simulation of the structural system or element in fire, is evaluated for each of the points in the sampling scheme. The sample space of the model variables, $x$ and the response, $y$ of the high-fidelity model forms the training data for the surrogate model.

\subsection{Model hypothesis and fitting}

A hypothesis for the surrogate model is assumed based on the complexity of the high-fidelity model. In the present study, a polynomial model is adopted. Eq. (2) illustrates a first order polynomial hypothesis $(m=1)$ to represent the surrogate model for the high-fidelity model.

$$
\widehat{y}=\widehat{h}(x)=\theta_{0}+\sum_{\mathrm{i}=1}^{\mathrm{r}} \theta_{\mathrm{i}} x_{\mathrm{i}}
$$

where, $\hat{y}=\hat{h}(\boldsymbol{x})$ represents the surrogate model approximation, and $\theta=\left[\theta_{0}, \theta_{1}, \ldots, \theta_{\mathrm{r}}\right]$ represents the regression coefficients, where $\theta_{0}$ refers to the bias term and $\theta_{1}$ to $\theta_{\mathrm{r}}$ refers to the weights of the respective polynomial terms of the surrogate model.

The regression coefficients of the surrogate model are obtained through minimization of the cost of prediction (i.e. the divergence between the high-fidelity evaluations and the surrogate predictions for the training samples) using an optimization algorithm. Here, a gradient descent technique is adopted [15]. The cost of prediction is here referred to as cost function, $J(\theta)$, which is given by:

$$
J(\theta)=\frac{1}{2 n} \sum_{i=1}^{n}\left(\hat{h}\left(\boldsymbol{x}_{\boldsymbol{i}}\right)-y_{i}\right)^{2}
$$

where $n$ refers to the size of the training sample. The gradient descent is an optimization algorithm following downhill approach, where regression coefficients are obtained iteratively in the reverse direction to the positive gradient of the cost function. The regression coefficients at each iteration of the optimization procedure are given by:

$$
\theta_{r}=\theta_{r}-\alpha \frac{\partial}{\partial \theta_{r}} J(\theta)
$$

where, $\alpha$ is the learning rate for the surrogate model optimization. The regression coefficients are changed iteratively in each step until the cost function reaches a specified tolerance value. Based on the estimated regression coefficients and polynomial surrogate model, i.e. Eq. (2), the response of the high-fidelity model can be predicted straightforwardly.

\subsection{Fitting issues, optimization of hyperparameters and performance evaluation}

In the procedure of Section 2.2, it can be difficult to achieve the recommended tolerance value for the cost function because the input variables have different orders of magnitude (and dimensions), which results in unequal weighting of the features during updating of regression coefficients. To address this issue, the features (model variables) need to be scaled before the regression optimization. In the present study, the features are scaled based on the standardization technique, where the normalized values are given by: 


$$
x_{\text {norm }}=\frac{x-\mu_{x}}{\sigma_{x}}
$$

where $x$ refers to a particular feature, while $\mu_{x}$ and $\sigma_{x}$ are the mean and standard deviation of the feature values used for training the model.

Another issue in training a regression model is underfitting or overfitting of data. This issue is related to the training parameters of the surrogate model, namely size of the training set (n) and order of polynomial (m). Adoption of a lower order polynomial as surrogate model hypothesis might lead to an underfitting of the model, while there is an overfitting of data if higher order polynomials are adopted. Likewise, an insufficient sample size is incapable of mapping the entire sample space of the model variables, leading to a surrogate model which is limited in application scope. Thus, an appropriate polynomial order and sample size are needed for training a surrogate model to achieve accurate response prediction. The issue of overfitting of a surrogate model can be addressed by adopting a regularized cost function as shown in Eq. (6), where the regularization parameter $\lambda$ penalizes the regression coefficients by artificially increasing the cost function if polynomials are adopted with more significant (non-zero) coefficients.

$$
J_{\text {learn }}(\theta)=\frac{1}{2 n} \sum_{i=1}^{n}\left(\hat{h}\left(\mathbf{x}_{\mathbf{i}}\right)-y_{i}\right)^{2}+\frac{\lambda}{2 n} \sum_{l=1}^{r} \theta_{l}^{2}
$$

In Eq. $6, J_{\text {learn }}(\theta)$ is the regularized cost function. However, it is necessary to determine a proper value of regularization parameter $(\lambda)$. Similarly, an appropriate (minimum) sample size $(n)$ and order of polynomial ( $m$ ) should also be chosen to address the issue as a whole. These parameters can also be called hyperparameters and can be determined through the development of 'learning curves'. In order to develop the learning curves, three input data sets are considered, namely a training set, a cross-validation set and a test set. The learning curves then refer to the plot of the cost-function evaluated from the training $\left(J_{\text {train }}\right)$ and cross-validation data set $\left(J_{\mathrm{cv}}\right)$, i.e.:

$$
\begin{gathered}
J_{\text {train }}(\theta)=\frac{1}{2 n_{\text {train }}} \sum_{i=1}^{n_{\text {train }}}\left(\hat{h}\left(\boldsymbol{x}_{\text {train }, i}\right)-y_{\text {train }, i}\right)^{2} \\
J_{c v}(\theta)=\frac{1}{2 n_{\mathrm{cv}}} \sum_{i=1}^{n_{c v}}\left(\hat{h}\left(\boldsymbol{x}_{c v, i}\right)-y_{c v, i}\right)^{2}
\end{gathered}
$$

The approach for estimating hyperparameter involves developing learning curves by varying training parameters, each at a time and evaluating the prediction errors. After, the desired accuracy has been achieved, the surrogate model is considered sufficiently trained to predict the response of the high-fidelity model. Finally, the accuracy of the developed surrogate model with the particular combination of hyperparameters is evaluated based on the test data set. Here, the coefficient of determination, $R^{2}[16]$ is evaluated for estimating the prediction efficiency of the surrogate model:

$$
R^{2}=1-R_{\text {res }} / R_{\text {tot }}
$$

where, $R_{\text {res }}=\sum_{i=1}^{n_{\text {test }}}\left(\hat{h}\left(\boldsymbol{x}_{\text {test }, i}\right)-y_{\text {test }, i}\right)^{2}$ and $R_{\text {tot }}=\sum_{i=1}^{n_{\text {test }}}\left(y_{\text {test }, i}-\bar{y}_{\text {test }}\right)^{2}$ refers to the residual and total sum of squares, respectively. The $R^{2}$ ranges from 0 to 1 , where ' 1 ' represents a perfectly fitted surrogate model.

\section{APPLICATION TO REINFORCED CONCRETE COLUMN}

In this study, the methodology for the development of a regression-based surrogate model is demonstrated by considering the assessment of the fire-resistance rating of a RC column. The high-fidelity model is a finite element model in SAFIR [17], which involves a computational cost to solve the nonlinear thermalstructural computation. The probabilistic studies have been presented in [18] and incorporated in [19]. The cross-section of the considered column is $500 \mathrm{~mm} \times 500 \mathrm{~mm}$, reinforced with 12 bars of $20 \mathrm{~mm}$ diameter, which is shown in Figure 1(a). The height of the column is $4 \mathrm{~m}$, with pinned support at the bottom, and roller support at the top (restraining lateral movement). The grade of the concrete used is $\mathrm{C} 30 / 37$ and the 
reinforcing steel is of grade 500. The column is subjected to the ISO834 fire from all sides. Figure 1(b-c) shows the thermal and mechanical response of the considered concrete column with an axial loading of $6000 \mathrm{kN}$. Based on Figure 1, the column is observed to have the fire-resistance of $140 \mathrm{~min}$.

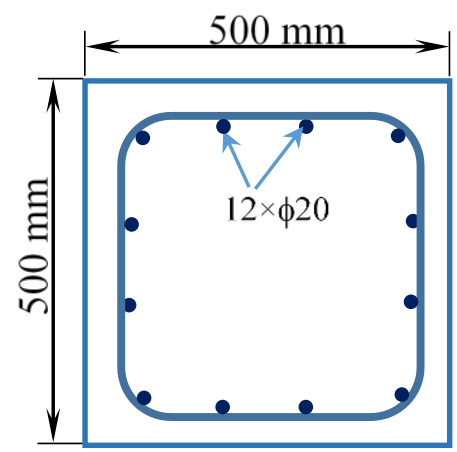

(a) Cross-section of column

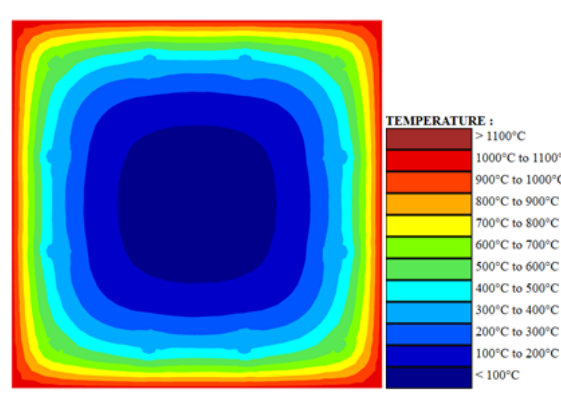

(b) Thermal analysis

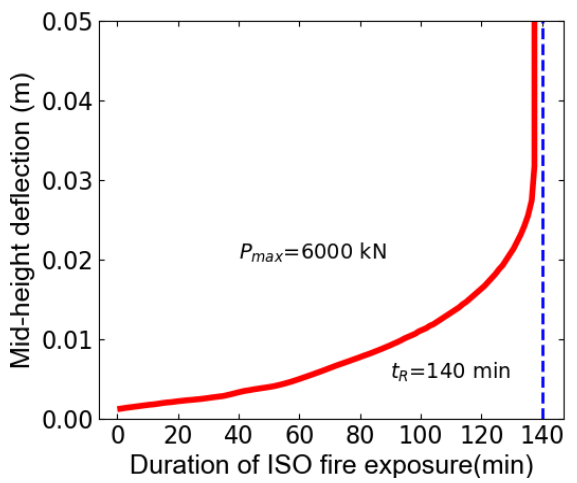

(c) Mechanical analysis

Figure 1. Response of RC column exposed to ISO834 fire, with an axial load of $6000 \mathrm{kN}$

\subsection{Development of surrogate model}

For the development of the surrogate model, the model variables for the high-fidelity model need to be selected first. For the RC column, seven variables are identified, namely: the retention factor parameters for the compressive strength of concrete $\left(\varepsilon_{\mathrm{kfc}}\right)$ and yield strength of reinforcing bars $\left(\varepsilon_{\mathrm{kfy}}\right)$, the concrete cover $(c)$, average eccentricity $(e)$, out of straightness $(f)$, out of plumbness $(\Phi)$ and the applied load $(P)$. The variables $\varepsilon_{\mathrm{kfc}}$ and $\varepsilon_{\mathrm{kfy}}$ define the quantile of the retention factors at elevated temperature based on the strength retention models by Qureshi et al. [20]. The model variables $e, f$ and $\Phi$ are the three eccentricities of the column, as listed in the JCSS probabilistic model code [21]. The considered model variables along with the ranges for the sample space are shown in Table 1. An LHS scheme is adopted for developing the sample space for training the surrogate model. The generated training data initially contains a training set of $10^{4}$ realizations (LHS set) and a cross-validation 1250 realizations (MC). Finally, the high-fidelity model is evaluated for each of these generated training and cross-validation sample points. In the present study, to allow comparison with the results by Van Coile et al. [18], the surrogate model is trained to predict the maximum axial load bearing capacity of the column $\left(P_{\max }\right)$ for a specified duration $(240 \mathrm{~min})$ of ISO 834 fire exposure. In order to achieve this, the numerically obtained fire resistance for the RC column is considered as input parameter for the surrogate model, while the axial load on the column is considered as response of the surrogate model.

Table 1. Model variables and their distribution for development of surrogate model

\begin{tabular}{lcccc}
\hline Independent variables & $\begin{array}{c}\text { Lower } \\
\text { limit }\end{array}$ & $\begin{array}{c}\text { Upper } \\
\text { limit }\end{array}$ & Unit & Sampling distribution \\
\hline Concrete strength retention factor parameter, $\varepsilon_{\mathrm{kfc}}$ & -4.00 & 4.00 & - & \\
\hline Rebar yield strength retention factor parameter, $\varepsilon_{\mathrm{kfy}}$ & -4.00 & 4.00 & - & \\
\hline Concrete cover, $c$ & 16 & 96 & $\mathrm{~mm}$ & \multirow{2}{*}{ Uniform } \\
\hline Average eccentricity, $e$ & -0.03 & 0.03 & $\mathrm{~mm}$ \\
\hline Out of straightness, $f$ & -0.03 & 0.03 & $\mathrm{~mm}$ \\
\hline Out of plumbness, $\Phi$ & -0.01 & 0.01 & $\mathrm{~mm}$ & \\
\hline Maximum applied load, $P_{\max }$ & 1000 & 10500 & $\mathrm{kN}$ \\
\hline
\end{tabular}

Next, the surrogate model hypothesis is defined and the model is trained based on the developed sample space and evaluated response from SAFIR analyses (the high-fidelity model). Subsequently, the learning curves are developed to assess the optimum value of the hyperparameters by evaluating $J_{\text {train }}$ and $J_{\mathrm{CV}}$ for different sets of the hyperparameters. Figure 2 shows the learning curves for the surrogate model of the RC column considered in the study, which allows to determine optimized hyperparameters. 
Figure 2 (a) allows to determine the optimum order of polynomial to be adopted for the surrogate model to work precisely. The $\mathbf{J}_{\mathrm{cv}}$ shows significant improvement in prediction error for polynomials with $m \leq 3$, while there is no further decrease in error for polynomial with $\mathrm{m} \geq 5$. Thus, a $4^{\text {th }}$ order polynomial hypothesis is adopted for the surrogate model hypothesis.

Similarly, Figure 2(b) helps to determine the optimum training sample size of the surrogate model. Based on the Figure, a training set of $10^{4}$ LHS samples is considered to be adequate to develop the surrogate model as the learning curves shows a sign of convergence. Figure 2(c) is developed considering estimated optimum order of polynomial and training sample size, where $J_{\text {train }}$ and $J_{\mathrm{cv}}$ are evaluated for varying regularization parameter values. The figure shows that the regularization parameter is not required for the considered surrogate model, as the cost function monotonously increases with increasing value of the regularization parameter. The learning curves indicate that $J_{t r a i n}$ is higher than $J_{C V}$. This may seem unexpected, but the LHS scheme adopted for the training set ensures sampling evenly across the entire input space, necessarily including many low probability (outlier) parameter combinations, while the MCS samples directly from the parameter distributions, without ensuring that also low probability combinations are included in the set. It is thus hypothesized that the higher $J_{\text {train }}$ results from a higher proportion of low "extreme" cases which are less well captured by the surrogate model.

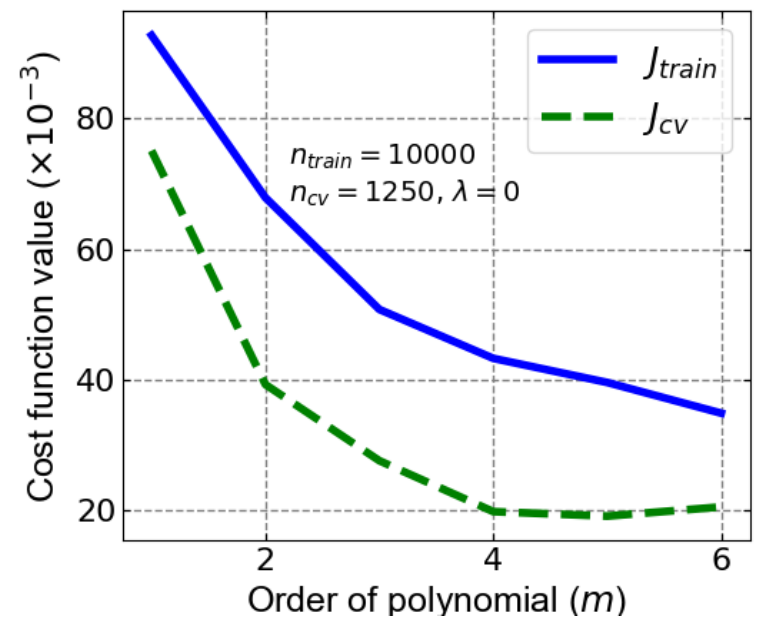

(a)

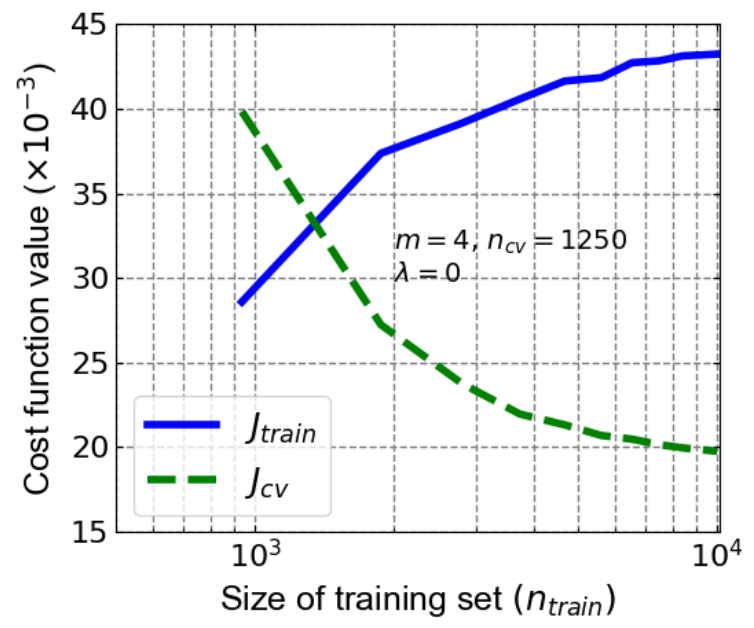

(b)

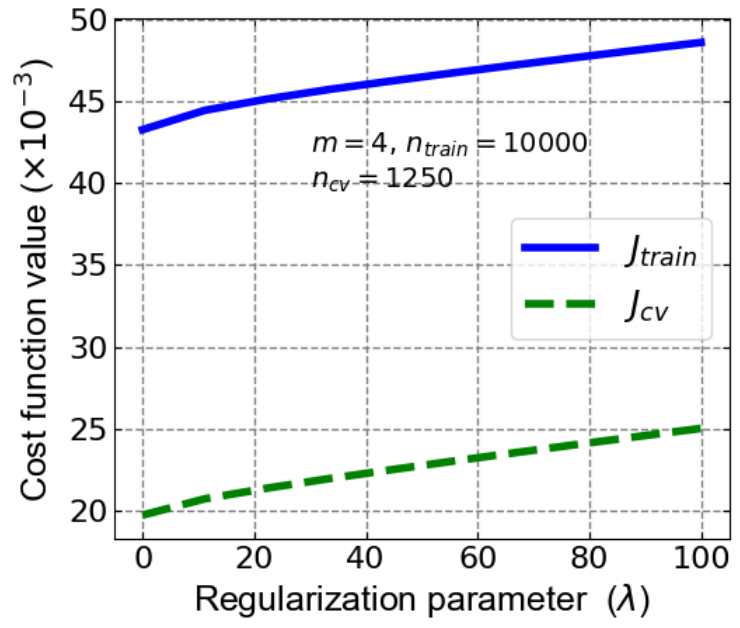

(c)

Figure 2. Learning curves for development of surrogate model

\subsection{Performance evaluation of surrogate model}

To evaluate the performance of the developed surrogate model in Section 3.1, a Monte-Carlo test set of 1250 sample points is considered. Figure 3(a) shows the comparison of the response for the test data set based on the high-fidelity model (i.e. SAFIR) and the surrogate model. The coefficient of determination $\left(R^{2}\right)$ for the surrogate model is found to be 0.95 . Similarly, Figure 3(b) shows the absolute normalized error 
(for the test data set, indicating that more than $90 \%$ of the test samples are predicted by the surrogate model with an error of less than 20\%. The absolute normalized error (ANE) for the test data set is given by:

$$
\mathrm{ANE}=\left|\frac{y_{\text {test }}-\hat{h}\left(\boldsymbol{x}_{\text {test }}\right)}{y_{\text {test }}}\right|
$$

A mean ANE value of 0.0506 is observed for the predicted capacity for the column. As the FE model for the RC column considered in the present study involves complex structural calculations, the evaluated error is considered reasonable. Thus, the surrogate model is implemented to predict the response of the RC column. The evaluation of the FE model for all the test samples took 60 core-hour on a state-of-the-art personal computer, while the evaluation is quasi-instantaneous for the surrogate model.

Considering the trained surrogate model, the regression coefficient for the duration of fire exposure $\left(t_{\mathrm{E}}\right)$ has the highest absolute value and thus can be considered as the most influencing parameter of the surrogate model. In contrast, there is negligible influence of the bias term on the response of surrogate model and thus this term can be neglected.

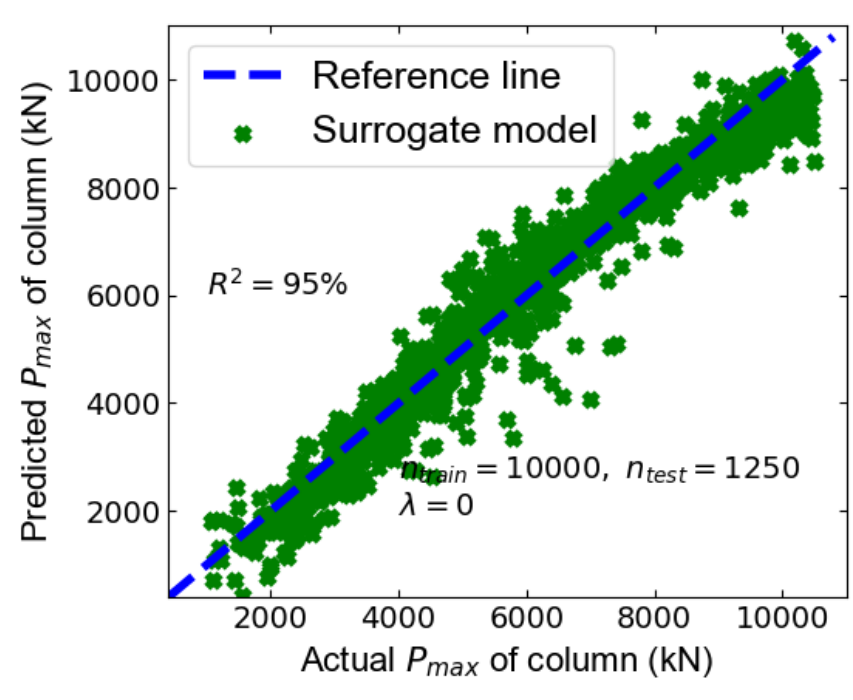

(a)

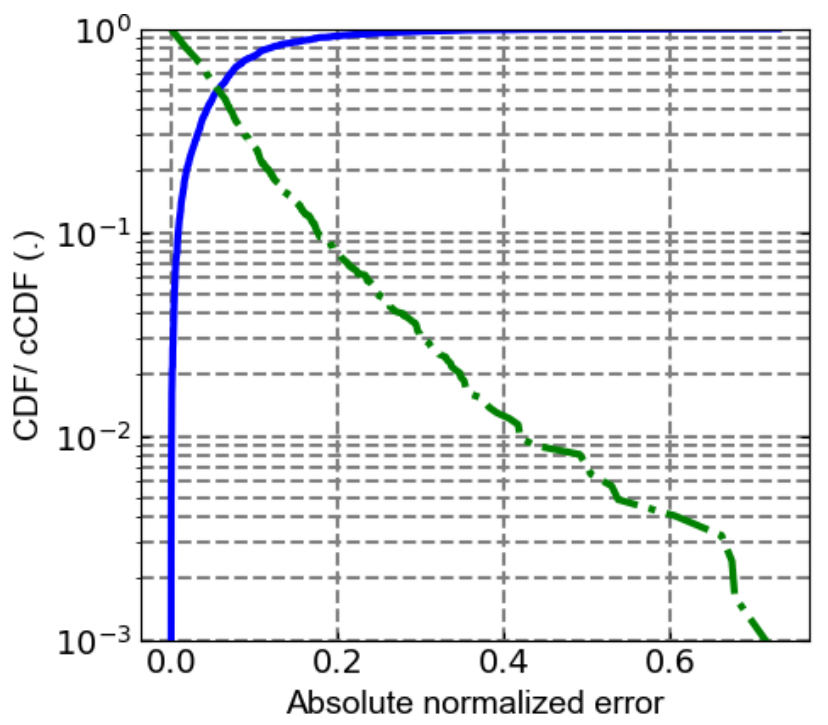

(b)

Figure 3. Performance of the surrogate model for the RC column. The output $P_{\max }$ is the column axial capacity after 240 min of ISO fire exposure. Actual value is obtained with SAFIR. Predicted value is obtained by the surrogate model.

\section{SURROGATE MODEL BASED PROBABILISTIC STUDIES}

Figure 4 shows the framework proposed for probabilistic studies of fire exposed structures based on the regression-based surrogate models. For the probabilistic studies, initially a surrogate model needs to be developed for the high-fidelity model (SAFIR model here). Section 2 above elaborates the steps for developing a surrogate model, which has been implemented in Section 3 for the RC column. Once the surrogate model with the desired accuracy is developed, the probabilistic distributions of the model variables can be considered for the specific design to carry out a probabilistic evaluation. In this regard, a sample space is developed based on the assumed probabilistic distribution through a sampling scheme (LHS considered here) and subsequently, the response of the structure is evaluated considering the surrogate model for each of the sample points. Finally, fragility curves are developed based on the estimated responses. As the surrogate model involves significantly lower computational cost, the probabilistic estimations can be done at limited computational expense, also considering design changes. 


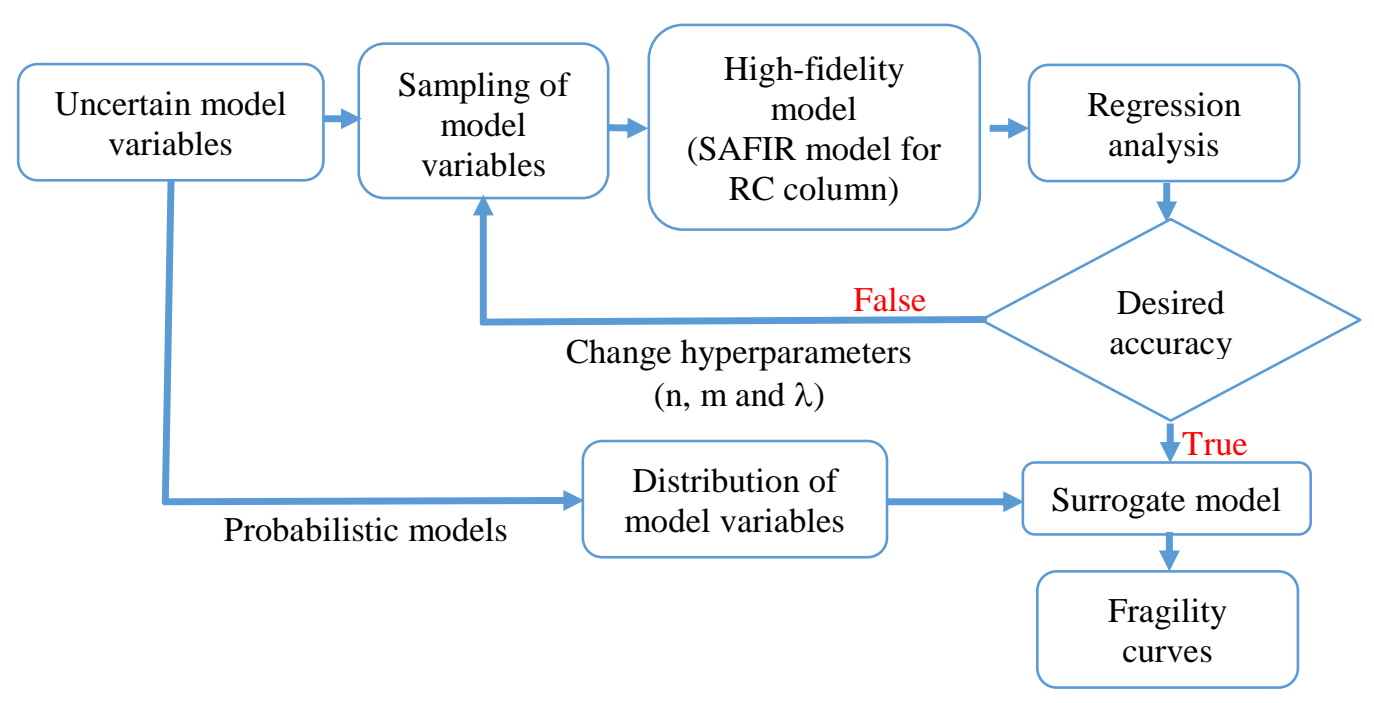

Figure 4. Framework for probabilistic studies of fire exposed structures based on surrogate modelling methodology

\subsection{Concept validation}

To validate the proof-of-concept, the probability density function (PDF) and cumulative density function (CDF) for the considered RC column, developed based on the proposed framework is compared against that obtained by a traditional direct Monte Carlo approach. The validation is carried out for maximum axial load for the considered RC column exposed to 4 hours of ISO834 fire, as presented in [18]. Table 2 shows the uncertain model variables along with their probabilistic distributions $[18,21]$ for the probabilistic evaluation. A set of $10^{4}$ LHS samples are developed for the considered stochastic variables. Finally, the $\mathrm{PDF}$ and CDF for the RC column can easily be evaluated based on the surrogate model developed in Section 2. On the other hand, the development of PDF and CDF based on the traditional Monte Carlos method involves direct evaluation of the complex high-fidelity model and is computationally expensive, notably due to a need to apply an iterative search algorithm to determine the maximum allowable axial load to achieve 240 min standard fire resistance for each RC column realization.

Table 2. Stochastic variables and their distributions for probabilistic evaluation of RC column

\begin{tabular}{cccc}
\hline Stochastic variables & Distribution & Mean & Standard deviation \\
\hline $\begin{array}{c}\text { Yield strength of rebars, } f_{\mathrm{y}, \mathrm{T}} \\
\left(f_{\mathrm{y}, 20}=500 \mathrm{MPa} ; \boldsymbol{\mu}_{f y, 20}=560 \mathrm{MPa}\right)\end{array}$ & Logistic model [20] & Temperature-dependent & Temperature-dependent \\
\hline $\begin{array}{c}\text { Concrete compressive strength, } f_{\mathrm{c}, \mathrm{T}} \\
\left(f_{c k, 20}=30 \mathrm{MPa} ; \boldsymbol{\mu}_{f y, 20}=42 \mathrm{MPa}\right)\end{array}$ & & & 5 \\
\hline Concrete cover, $\boldsymbol{c}[\mathbf{m m}]$ & Beta $[\mu-3 \sigma ; \mu+3 \sigma]$ & $42+5=47$ & 0.001 \\
\hline Average eccentricity, $\boldsymbol{e}[-]$ & Normal & 0 & 0.001 \\
\hline Out of straightness, $\boldsymbol{f}[-]$ & Normal & 0 & 0.0015 \\
\hline Out of plumbness, $\boldsymbol{\Phi}[-]$ & Gumbel & 0 & 5 \\
\hline
\end{tabular}

Figure 5 shows the comparison of the PDF and CDF for the RC column, evaluated based on direct estimation from the high-fidelity model $\left(10^{4}\right.$ evaluations) and the surrogate model. Based on Figure 5(a), the PDF are quite in agreement. The mean predicted value for the load capacity of the column for 4 hours of ISO834 exposure is $5137 \mathrm{kN}$ based on the surrogate model, which is quite close to the actual capacity of $5038 \mathrm{kN}$, evaluated through the SAFIR Monte-Carlo approach. Likewise, Based on Figure 5(b), it can be determined that the CDF based on application of the surrogate model agrees reasonably well with the CDF evaluated through SAFIR Monte Carlo computations, especially with respect to the lower quantile of $P_{\max }$. The comparison of the capacity quantiles is shown in Table 3, where a prediction error of less than $5 \%$ can be observed. In the Table, the $10^{-2}$ capacity quantiles for RC column based on actual model is $2931 \mathrm{kN}$, which is predicted as $3010 \mathrm{kN}$ by the surrogate model, indicating an error of $2.7 \%$. 
Table 3. Capacity quantiles for load capacity of RC column exposed to ISO 834 fire

\begin{tabular}{ccccc} 
S.N & CDF (.) & \multicolumn{3}{c}{ MR of slab (kN) for RC column } \\
\cline { 3 - 5 } & & $\begin{array}{c}\text { Surrogate model } \\
(M L)\end{array}$ & $\begin{array}{c}\text { Actual model } \\
(\text { SAFIR })\end{array}$ & ANE $(\%)$ \\
\hline 1. & $10^{-1}$ & 3987 & 3805 & 4.7 \\
\hline 2. & $10^{-2}$ & 3010 & 2931 & 2.70 \\
\hline 3. & $10^{-3}$ & 2475 & 2414 & 2.52 \\
\hline
\end{tabular}

These results suggest that the surrogate modelling methodology can be used for probabilistic studies of fire exposed structures (provided adequate training data has been generated), thereby validating the proof-ofconcept. From the perspective of computational cost, the probabilistic estimation of capacity of column based on the trained surrogate model is instantaneous, while the same evaluation required approximately 7 days through a traditional Monte-Carlo approach. Thus, the surrogate modelling methodology being computationally efficient is considered promising for the probabilistic studies of fire exposed structures.

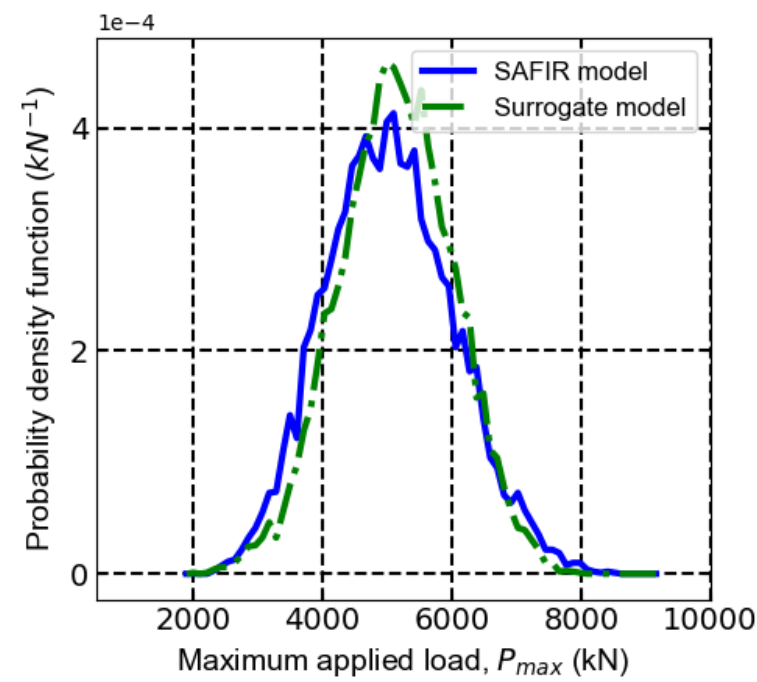

(a)

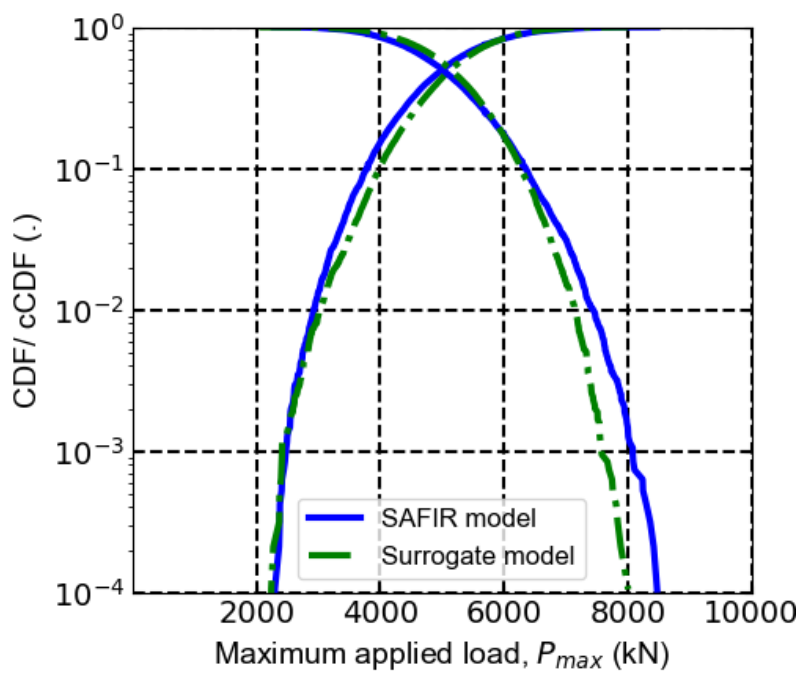

(b)

Figure 5. PDF and CDF for the concrete column based on actual and surrogate model

\subsection{Generalized fragility curves}

The direct Monte-Carlo approach for the probabilistic evaluation involves an iterative evaluation of the high-fidelity system in order to evaluate $\mathrm{P}_{\max }$ for a given ISO834 fire duration, which is a computationally expensive task. Thus, it is impractical to perform probabilistic evaluations in case of a change in the design variables. In this regard, the surrogate model can be most helpful as it allows quasi-instantaneous prediction of the structural response for any alteration in design variables (within the parameter range for which the model has been trained). This concept is demonstrated through the application of the developed surrogate model to different ISO834 exposure durations in Figure 6. Based on the evaluations, the mean load carrying capacities of the RC column for 1, 2, 3 and 4 hours of ISO834 exposure are $8547 \mathrm{kN}, 7290 \mathrm{kN}, 6210 \mathrm{kN}$ and 5137 $\mathrm{kN}$, respectively. The $10^{-2}$ capacity quantiles for the RC column are predicted as $6513 \mathrm{kN}, 4944 \mathrm{kN}, 3852 \mathrm{kN}$ and $3010 \mathrm{kN}$, respectively. The results of Figure 6 for $P_{\max }$, can be understood as fragility curves, where the intensity measure is the maximum applied load on the column. This allows an engineer to swiftly evaluate the probability of failure of the column at different times of exposure, given its applied load in the fire situation. For example, if the $\mathrm{RC}$ column is subjected to an applied load of $4500 \mathrm{kN}$, the failure probability under ISO exposure is $0.3 \%$ after $2 \mathrm{~h}$, $4.30 \%$ after $3 \mathrm{~h}$, and $24.53 \%$ after $4 \mathrm{~h}$. The failure probability is virtually null after $1 \mathrm{~h}$. These fragility curves can be generated efficiently for any alteration in design parameters through the surrogate modelling methodology. 


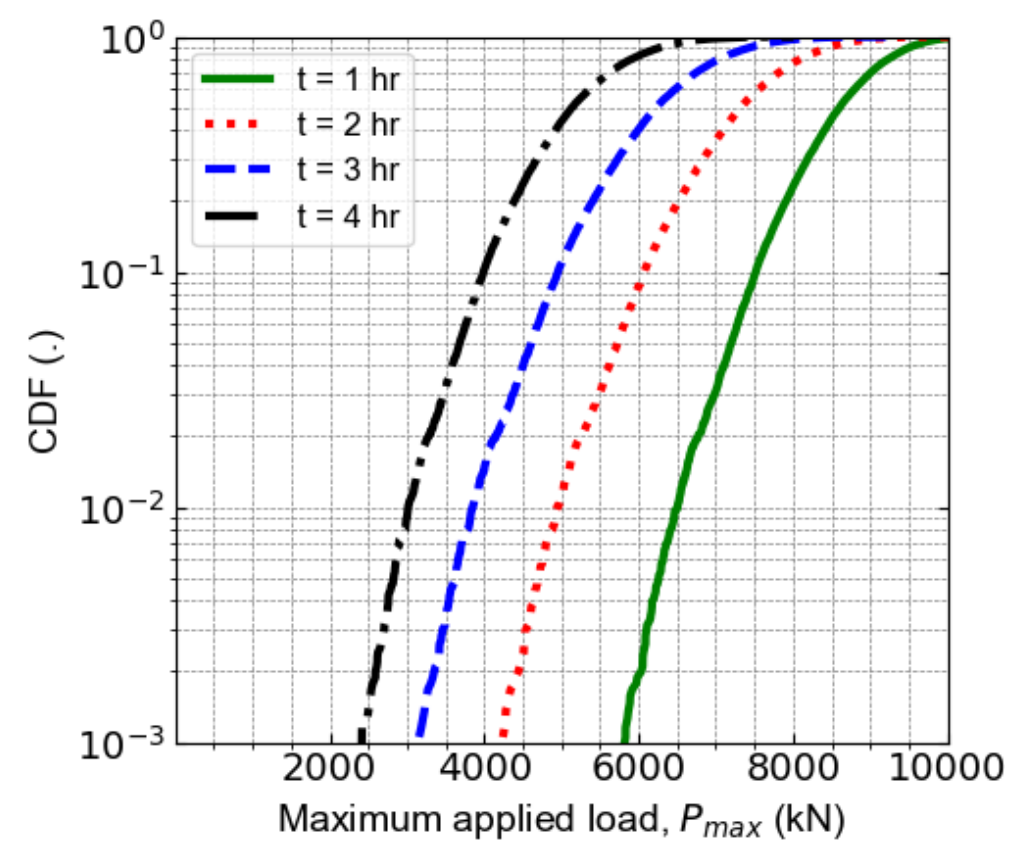

Figure 6. CDF for the RC column exposed to ISO834 fire of different durations

\section{CONCLUSIONS}

Available prescriptive guidelines and code-based recommendations are at times insufficient for the design of structures under fire exposure. In certain cases, the use of probabilistic approaches, where the important uncertainties in the model evaluation are taken into account, is required to explicitly assess the risk profile of the fire hazard on structures. However, probabilistic evaluations are complex and computationally demanding, and as most structural fire analysis involves complex structural calculations, it is impractical to adopt direct probabilistic approaches. To address this issue, this study has proposed adopting regressionbased surrogate models for the probabilistic evaluation of fire exposed structures. The surrogate models can simulate the behaviour of a complex high-fidelity model precisely and can predict the response of the model quasi-instantaneously. The current study acts as a proof-of-concept.

In the present study, the basic case of a fire exposed reinforced concrete (RC) column is considered. Initially, a surrogate model was developed for the $\mathrm{RC}$ column, which can predict the maximum load carrying capacity for a given ISO834 exposure duration. The coefficient of determination for the surrogate model was found to be 0.95 . The developed surrogate model was then adopted for probabilistic studies, where the probability density functions (PDF) and cumulative distribution functions (CDF) were evaluated. These PDF and CDF are quite in agreement with those obtained through the direct evaluation of the computationally more expensive high-fidelity model. The $0.1 \%, 1 \%$ and $10 \%$ capacity quantiles show a maximum error of $5 \%$. The probabilistic study based on the surrogate model is quasi-instantaneous while the direct estimation through a Monte Carlo approach involves significant computational cost (approximately 7 days). This observation makes it possible for design iterations (changes in design parameters) to be considered in a probabilistic analysis, harnessing the surrogate modelling approach. Thus, based on the current study, surrogate modelling seems a promising methodology to advance probabilistic studies in the field of structural fire engineering, allowing computationally efficient probabilistic evaluations during structural fire design.

\section{REFERENCES}

1. Van Coile, R., Hopkin, D., Lange, D., Jomaas, G., and Bisby, L. (2019). The need for hierarchies of acceptance criteria for probabilistic risk assessments in fire engineering. Fire Technology, 55(4), 1111-1146.

2. Gernay, T., Van Coile, R., Khorasani, N.E. and Hopkin, D., (2019a). Efficient uncertainty quantification method applied to structural fire engineering computations. Engineering Structures, 183, 1-17. 
3. Shrivastava, M., Abu, A. K., Dhakal, R. P., and Moss, P. J. (2019). Severity measures and stripe analysis for probabilistic structural fire engineering. Fire Technology, 55(4), 1147-1173.

4. Heidari, M., Robert, F., Lange, D. and Rein, G. (2019). Probabilistic study of the resistance of a simplysupported reinforced concrete slab according to eurocode parametric fire. Fire Technology, 55(4), pp.13771404.

5. Gernay, T., Khorasani, N.E. and Garlock, M., (2019b). Fire fragility functions for steel frame buildings: sensitivity analysis and reliability framework. Fire Technology, 55(4), pp.1175-1210.

6. Van Coile, R., Caspeele, R., and Taerwe, L. (2014). Reliability-based evaluation of the inherent safety presumptions in common fire safety design. Engineering structures, 77, 181-192.

7. Guo, Q., Shi, K., Jia, Z., and Jeffers, A. E. (2013). Probabilistic evaluation of structural fire resistance . Fire technology, 49(3), 793-811.

8. Hopkin, D., Van Coile, R., and Fu, I. (2018). Developing fragility curves and estimating failure probabilities for protected steel structural elements subject to fully developed fires. Proceedings of the 10th International Conference on Structures in Fire. 06-08/06, Belfast, UK.

9. Forrester, A., Sobester, A. and Keane, A., (2008). Engineering design via surrogate modelling: a practical guide. John Wiley \& Sons.

10. Dexters A., Leisted R. R., Van Coile R., Welch S., Jomaas, G. (2019). Testing for Knowledge: Maximising Information Obtained from Fire Tests by using Machine Learning Techniques. Proceedings of the 15th International Interflam conference, 1.

11. Seitllari, A. and Naser, M.Z. (2019). Leveraging artificial intelligence to assess explosive spalling in fireexposed RC columns. Comput. Concr, 24, pp.271-282.

12. Naser, M. Z., (2019). Fire resistance evaluation through artificial intelligence-A case for timber structures. Fire safety journal, 105, 1-18.

13. James, G., Witten, D., Hastie, T. and Tibshirani, R. (2013). An introduction to statistical learning. New York: springer, 112, 3-7.

14. Olsson, A., Sandberg, G. and Dahlblom, O. (2003). On Latin hypercube sampling for structural reliability analysis. Structural safety, 25(1), 47-68.

15. Du, S.S., Lee, J.D., Li, H., Wang, L. and Zhai, X. (2018). Gradient descent finds global minima of deep neural networks. arXiv preprint arXiv:1811.03804.

16. Draper, N. R. and Smith, H. (1998). Applied Regression Analysis. Wiley-Interscience, 326.

17. Franssen, J.M. and Gernay, T., 2017. Modeling structures in fire with SAFIR®: Theoretical background and capabilities. Journal of Structural Fire Engineering, 8(3), 300-323.

18. Van Coile, R., Hopkin, D., Elhami Khorasani, N. and Gernay, T., (2020). Demonstrating adequate safety for a concrete column exposed to fire, using probabilistic methods. Fire and Materials. https://doi.org/10.1002/fam.2835.

19. ISO/CD TR 24679-8: 2020. Fire safety engineering — Performance of structures in fire — Part 8: Example of a probabilistic fire design of structures. ISO Technical Report. International Organization for Standardization, Geneva, Switzerland.

20. Qureshi, R., Ni, S., Elhami Khorasani, N., Van Coile, R., Hopkin, D. and Gernay, T., (2020). Probabilistic Models for Temperature-Dependent Strength of Steel and Concrete. Journal of Structural Engineering, 146(6), 04020102.

21. JCSS. (2013). Probabilistic Model Code. Joint Committee on Structural Safety. Available online at http://www.jcss.byg.dtu.dk/. 